PROCEEDINGS OF THE

AMERICAN MATHEMATICAL SOCIETY

Volume 128, Number 10, Pages 2967-2972

S 0002-9939(00)05388-0

Article electronically published on April 7, 2000

\title{
STRONG CONNECTEDNESS OF THE INVERTIBLES IN A FINITE SUBDIAGONAL ALGEBRA
}

\author{
MICHAEL MARSALLI AND GRAEME WEST
}

(Communicated by David R. Larson)

\begin{abstract}
Suppose $H^{\infty}$ is a finite, subdiagonal subalgebra of a von Neumann algebra. We show that the invertible group of $H^{\infty}$ is strongly connected.
\end{abstract}

\section{INTRODUCTION}

Questions of connectedness are important in the theory of operator algebras. It is well known that the invertible group of a von Neumann algebra is path connected in the norm topology. On the other hand, this is a major open question in the theory of nest algebras. Moreover, it is known that in the classical Hardy space $H^{\infty}$ the invertibles are not norm connected. See for example 3. Chapter 25]. The algebra $H^{\infty}$ is the classical and motivating example for a wide class of operator algebras, introduced by Arveson, called finite subdiagonal algebras. (See the definitions and references below.) Thus one has that the invertible group of a finite subdiagonal algebra is not necessarily norm connected. In this paper we establish that the invertible group is in fact strongly connected.

The contrast between finite subdiagonal algebras and nest algebras is quite illuminating. Knowles and Saeks 9] established the strong connectedness of the invertibles in nest subalgebras of von Neumann algebras. But the deepest results about connectedness in nest algebras are due to Davidson, Orr, and Pitts. In [4] Davidson and Orr established norm connectedness of the invertibles for nest algebras whose atoms are all of infinite rank. Subsequently, Davidson, Orr, and Pitts [5] extended this result to nest algebras whose nest has no isolated atoms of finite multiplicity. Consequently, the norm connectedness question for nest algebras reduces to nests with atoms ordered as $\mathbb{Z}$ or $\mathbb{N}$. In these two recalcitrant cases, the strong connectedness is fairly easy to establish. But as we have seen, norm connectedness does not hold for all finite subdiagonal algebras. So strong connectedness is in a sense the best possible result.

We first establish some notation. Throughout $\mathcal{M}$ is a von Neumann algebra equipped with a faithful, normal finite tracial state $\tau$. The set of self-adjoints in $\mathcal{M}$ is denoted by $\mathcal{M}^{s a}$, the unitaries by $\mathcal{M}^{u}$, and the orthogonal projections by $\mathcal{M}^{p}$. The group of invertibles will be denoted $\mathcal{G}(\mathcal{M})$.

Received by the editors June 15, 1998 and, in revised form, November 22, 1998.

2000 Mathematics Subject Classification. Primary 46L52.

Key words and phrases. von Neumann algebra, finite subdiagonal algebras.

(C)2000 American Mathematical Society 
Definition 1 (cf. 2], [12, 11]). Let $\mathcal{M}$ be a von Neumann algebra with a faithful, normal finite tracial state $\tau$. Let $H^{\infty}$ be a weak*-closed unital subalgebra of $\mathcal{M}$, and let $\Phi$ be a faithful, normal expectation from $\mathcal{M}$ onto the diagonal von Neumann algebra $\mathcal{D}=H^{\infty} \cap\left(H^{\infty}\right)^{*}$. Then $H^{\infty}$ is a finite, subdiagonal subalgebra of $\mathcal{M}$ with respect to $\Phi$ if:

(1) $H^{\infty}+\left(H^{\infty}\right)^{*}$ is weak*-dense in $\mathcal{M}$,

(2) $\Phi(x y)=\Phi(x) \Phi(y)$ for all $x, y \in H^{\infty}$, and

(3) $\tau \circ \Phi=\tau$.

For examples and further references see [11. The group of invertibles will be denoted $\mathcal{G}\left(H^{\infty}\right)$. Also we let $H_{0}^{\infty}=\left\{h \in H^{\infty}: \Phi(h)=0\right\}$.

For $1 \leq p \leq \infty$, let $L^{p}(\mathcal{M})$ denote the noncommutative Lebesgue space which is associated with $\mathcal{M}$ and $\tau$ (see [7]). Closure in $L^{p}(\mathcal{M})$ will be denoted by $[\cdot]_{p}$. Let $H^{p}=\left[H^{\infty}\right]_{p}$ and $H_{0}^{p}=\left[H_{0}^{\infty}\right]_{p} . L^{p}(\mathcal{M})$ and $H^{p}$ are Banach spaces satisfying all hoped for properties such as duality.

Significantly, much attention has been focused on a type of result that can be called a unitary-analytic factorization. Roughly speaking, this is a factorization $x=u a$ of a given operator $x$ into the product of a unitary operator $u$ and an analytic $a$ where the $L^{p}(\mathcal{M})$ properties of $x$ are 'transferred' to $H^{p}$ properties of $a$. For example, Arveson showed that if $x \in \mathcal{G}(\mathcal{M})$, then there is such a factorization with $a \in \mathcal{G}\left(H^{\infty}\right)$ [2] Theorem 4.2.1]. Later this result was refined to show that if $x \in \mathcal{M}$ and $x^{-1}$ exists in $L^{2}(\mathcal{M})$, then a factorization exists with $a \in H^{\infty}$ and $a^{-1} \in H^{2}$ [12, Proposition 1.2], [14, Proposition 1]. In [10] and [11] such factorizations for $x \in L^{p}(\mathcal{M})$ were established.

All of these results are interesting in their own right but moreover have proved of fundamental technical importance in the evolution of the theory of finite subdiagonal algebras. For example, Arveson used his factorization to show that $H^{\infty}$ is logmodular [2 Corollary 4.2.4].

Of course these unitary-analytic factorizations are selections. In this paper we will demonstrate, by an analysis and refinement of the results and proofs in [2], [12] and [14] that the factorizations can be selected in such a manner that both the unitary component and the analytic component are continuous arguments of the original operator. Then we show that the invertibles of $H^{\infty}$ are $L^{2}(\mathcal{M})$ connected and strongly connected.

\section{Continuous Selection in the Unitary-AnAlytic FaCtorization}

Definition $2([2, \S 4]) \cdot w \in L^{2}(\mathcal{M})$ is said to be right wandering if $\langle w f, w\rangle=0$ for every $f \in H_{0}^{\infty}$.

It is noted in 2] that if $w$ is right wandering, then $\left\langle w f^{*}, w\right\rangle=\tau\left(w^{*} w f^{*}\right)=$ $\overline{\tau\left(f w^{*} w\right)}=\overline{\tau\left(w^{*} w f\right)}=\overline{0}=0$ for every $f \in H_{0}^{\infty}$. It follows from ultraweak continuity that if $w$ is right wandering, then $\langle w s, w\rangle=\langle w \Phi(s), w\rangle$ for every $s \in \mathcal{M}$.

In the proof of [2] Lemma 4.2.2] it is shown that given any right wandering $w$, there exists $v \in L^{2}(\mathcal{D})$ such that $\langle w d, w\rangle=\langle v d, v\rangle$ for all $d \in \mathcal{D}$. It is easy to see that $v$ is not uniquely determined. However, since $\langle v d, v\rangle=\left\langle v^{*} v d, 1\right\rangle=\left\langle|v|^{2} d, 1\right\rangle=$ $\langle|v| d,|v|\rangle$, we may in fact replace $v$ with $|v|$. Also, we still have $|v| \in L^{2}(\mathcal{D})$, as required. Furthermore, with the positivity condition, $v$ is uniquely determined. To see this, suppose $0 \leq v_{1}, v_{2} \in L^{2}(\mathcal{D})$ satisfy $\left\langle v_{1} d, v_{1}\right\rangle=\langle w d, w\rangle=\left\langle v_{2} d, v_{2}\right\rangle$ for all $d \in \mathcal{D}$. Then $v_{1}^{2}=v_{2}^{2}$, since $\mathcal{D}$ separates the points of $L^{1}(\mathcal{D})$, and so $v_{1}=v_{2}$, by uniqueness of positive square roots. 
Theorem 1. Suppose $w$ is right wandering and $0 \leq v \in L^{2}(\mathcal{D})$ is chosen as above. Then the map $w \rightarrow v$ is continuous in $L^{2}(\mathcal{M})$.

Proof. Suppose $\epsilon>0$ and suppose $w_{1}$ is right wandering. Choose $\delta>0$ so that $\left(2\left\|w_{1}\right\|_{2}+\delta\right) \delta<\epsilon^{2}$. Suppose $w_{2}$ is also right wandering and that $\left\|w_{1}-w_{2}\right\|_{2} \leq \delta$. As has already been established, there exist $v_{i} \in L^{2}(\mathcal{D}), v_{i} \geq 0$ such that $\left\langle w_{i} d, w_{i}\right\rangle=$ $\left\langle v_{i} d, v_{i}\right\rangle$ for $d \in \mathcal{D}$ and $i=1,2$. To prove the theorem we show that $\left\|v_{1}-v_{2}\right\|_{2} \leq \epsilon$.

Firstly, note that $\left\|v_{1}-v_{2}\right\|_{2} \leq\left\|v_{1}^{2}-v_{2}^{2}\right\|_{1}^{1 / 2}$. This is the Powers-Størmer inequality [13], or can be seen as a consequence of a submajorisation result of Ando [1], which has been established in the most general setting [6].

Now for $d \in \mathcal{D}$ we have $\tau\left(\left[v_{1}^{2}-v_{2}^{2}\right] d\right)=\left\langle v_{1} d, v_{1}\right\rangle-\left\langle v_{2} d, v_{2}\right\rangle=\left\langle w_{1} d, w_{1}\right\rangle-$ $\left\langle w_{2} d, w_{2}\right\rangle=\tau\left(\left[w_{1}^{*} w_{1}-w_{2}^{*} w_{2}\right] d\right)$. Thus, by Köthe duality, first applied to the pair $L^{1}(\mathcal{D}), \mathcal{D}$ and then to the pair $L^{1}(\mathcal{M}), \mathcal{M}$, we have

$$
\begin{aligned}
\left\|v_{1}^{2}-v_{2}^{2}\right\|_{1} & =\sup _{d \in B_{\mathcal{D}}}\left|\tau\left(\left[v_{1}^{2}-v_{2}^{2}\right] d\right)\right| \\
& =\sup _{d \in B_{\mathcal{D}}}\left|\tau\left(\left[w_{1}^{*} w_{1}-w_{2}^{*} w_{2}\right] d\right)\right| \\
& \leq \sup _{d \in B_{\mathcal{M}}}\left|\tau\left(\left[w_{1}^{*} w_{1}-w_{2}^{*} w_{2}\right] d\right)\right| \\
& =\left\|w_{1}^{*} w_{1}-w_{2}^{*} w_{2}\right\|_{1} .
\end{aligned}
$$

Now from Hölder's inequality we have that

$$
\left\|w_{1}^{*} w_{1}-w_{2}^{*} w_{2}\right\|_{1} \leq\left(\left\|w_{1}\right\|_{2}+\left\|w_{2}\right\|_{2}\right)\left\|w_{1}-w_{2}\right\|_{2} \leq\left(2\left\|w_{1}\right\|_{2}+\delta\right) \delta \leq \epsilon^{2} .
$$

It follows that $\left\|v_{1}-v_{2}\right\|_{2} \leq \epsilon$.

The following is a synopsis of results that may be obtained from a careful reading of the proofs of [2] Lemma 4.2.2 and Theorem 4.2.1]. See also [12, Proposition 1.2] and [14, Proposition 1].

Theorem 2. Suppose $x \in \mathcal{G}(\mathcal{M})$. Let $P_{x}$ be the orthogonal projection of $L^{2}(\mathcal{M})$ onto the closed subspace $x H_{0}^{2}$. Let $w:=\left(I-P_{x}\right) x$. Then

(a) $w \neq 0$, w is right wandering, and $[w \mathcal{M}]_{2}=L^{2}(\mathcal{M})$.

(b) Given any right wandering $w$ there exists $v \in L^{2}(\mathcal{D})$ such that $\langle w d, w\rangle=$ $\langle v d, v\rangle$ for all $d \in \mathcal{D}$.

(c) Given such $x, w$ and $v$ there exists $u \in \mathcal{M}^{u}$ such that $u w=v, a:=u x \in$ $\mathcal{G}\left(H^{\infty}\right)$. Hence $x=u^{*}$ a is a unitary-analytic factorization.

(d) If $u_{1} a_{1}=x=u_{2} a_{2}$ are two such factorizations, then $u_{2}^{*} u_{1}=a_{2} a_{1}^{-1} \in \mathcal{D}^{u}$.

Remark 1 ([8] Remark 3.1.7]). Suppose $x \in \mathcal{G}(\mathcal{M})$. Let $\gamma(x)$ denote the minimum modulus of $x$. If $\|x-y\|_{\infty}<\frac{\gamma(x)}{2}$, then $y \in \mathcal{G}(\mathcal{M})$ and

$$
\left\|x^{-1}-y^{-1}\right\|_{\infty} \leq \frac{2}{\gamma(x)^{2}}\|x-y\|_{\infty} .
$$

In particular, $\left\|y^{-1}\right\|_{\infty} \leq \frac{2}{\gamma(x)^{2}}\|x-y\|_{\infty}+\left\|x^{-1}\right\|_{\infty}$.

Proposition 1. For $x \in \mathcal{G}(\mathcal{M})$ let $P_{x}$ be the orthogonal projection of $L^{2}(\mathcal{M})$ onto $x H_{0}^{2}$. Then the map $\left(\mathcal{G}(\mathcal{M}),\|\cdot\|_{\infty}\right) \rightarrow L^{2}(\mathcal{M}): x \rightarrow w:=\left(I-P_{x}\right) x$ is continuous.

Proof. Suppose $\epsilon>0$ and $x \in \mathcal{G}(\mathcal{M})$ are given. Choose $\delta>0$ so that $\delta \leq \frac{\gamma(x)}{2}$, $\delta \leq \frac{\gamma(x)^{2}}{2}, \delta \leq \frac{\epsilon}{4}, \delta\|x\|_{\infty}\left(\left\|x^{-1}\right\|_{\infty}+1\right) \leq \frac{\epsilon}{8}$ and $\delta\|x\|_{\infty}^{2}\left(\left\|x^{-1}\right\|_{\infty}+1\right) \leq \frac{\epsilon^{2}}{32}$.

Suppose $\|x-y\|_{\infty}<\delta$. For notational convenience let $P=P_{x}$ and $Q=P_{y}$. We show that $\|(I-P) x-(I-Q) y\|_{2} \leq \epsilon$. Let $P x=x h$ and $Q x=y f$ for some 
$h, f \in H_{0}^{2}$. Note that:

$$
\begin{aligned}
& \|x-x h\|_{2}=\|(I-P) x\|_{2} \leq\|x\|_{2} \leq\|x\|_{\infty} ; \\
& \|x-y f\|_{2}=\|(I-Q) x\|_{2} \leq\|x\|_{2} \leq\|x\|_{\infty} ; \\
& \|h\|_{2}=\left\|x^{-1} x h\right\|_{2} \leq\left\|x^{-1}\right\|_{\infty}\|x h\|_{2}=\left\|x^{-1}\right\|_{\infty}\|P x\|_{2} \leq\left\|x^{-1}\right\|_{\infty}\|x\|_{\infty} ; \\
& \|f\|_{2}=\left\|y^{-1} y f\right\|_{2} \leq\left\|y^{-1}\right\|_{\infty}\|y f\|_{2} \leq\left\|y^{-1}\right\|_{\infty}\|x\|_{\infty} \leq\left(\left\|x^{-1}\right\|_{\infty}+1\right)\|x\|_{\infty} ; \\
& \|x h-y h\|_{2} \leq\|x-y\|_{\infty}\|h\|_{2} \leq \delta\left\|x^{-1}\right\|_{\infty}\|x\|_{\infty} ; \\
& \|y f-x f\|_{2} \leq\|y-x\|_{\infty}\|f\|_{2} \leq \delta\left(\left\|x^{-1}\right\|_{\infty}+1\right)\|x\|_{\infty} .
\end{aligned}
$$

Now, using at the first step the Pythagorean relationship, we have

$$
\begin{aligned}
& \|x-y f\|_{2}^{2}+\|y f-y h\|_{2}^{2}=\|x-y h\|_{2}^{2} \\
\leq & {\left[\|x-x h\|_{2}+\|x h-y h\|_{2}\right]^{2} } \\
\leq & \|x-x h\|_{2}^{2}+2 \delta\|x\|_{\infty}^{2}\left\|x^{-1}\right\|_{\infty}+\delta^{2}\left\|x^{-1}\right\|_{\infty}\|x\|_{\infty} \\
\leq & \|x-x h\|_{2}^{2}+\frac{\epsilon^{2}}{32} \\
\leq & \|x-x f\|_{2}^{2}+\frac{\epsilon^{2}}{32} \\
\leq & {\left[\|x-y f\|_{2}+\|y f-x f\|_{2}\right]^{2}+\frac{\epsilon^{2}}{32} } \\
\leq & \|x-y f\|_{2}^{2}+2 \delta\|x\|_{\infty}^{2}\left(\left\|x^{-1}\right\|_{\infty}+1\right)+\delta^{2}\left(\left\|x^{-1}\right\|_{\infty}+1\right)^{2}\|x\|_{\infty}^{2}+\frac{\epsilon^{2}}{32} \\
\leq & \|x-y f\|_{2}^{2}+\frac{\epsilon^{2}}{16} .
\end{aligned}
$$

Here the fact that $\|x-x h\|_{2} \leq\|x-x f\|_{2}$ is a consequence of the best approximation property in Hilbert space. It follows that $\|y f-y h\|_{2} \leq \frac{\epsilon}{4}$. Hence

$$
\begin{aligned}
\|(I-P) x-(I-Q) y\|_{2} & =\|x-y+Q y-Q x+y f-y h+y h-x h\|_{2} \\
& \leq 2\|x-y\|_{2}+\|y f-y h\|_{2}+\|y h-x h\|_{2} \\
& \leq 2: \frac{\epsilon}{4}+\frac{\epsilon}{4}+\frac{\epsilon}{4} \\
& =\epsilon .
\end{aligned}
$$

Theorem 3. Let $x, w, v$ and $u$ be as in Theorem 2, but with $v$ chosen as in Theorem 1. Then the implied map $x \rightarrow u$ is continuous from $\left(\mathcal{G}(\mathcal{M}),\|\cdot\|_{\infty}\right)$ to $L^{2}(\mathcal{M})$.

Proof. Suppose $\epsilon>0$. Suppose $x_{1} \in \mathcal{G}(\mathcal{M})$ is given and that $w_{1}, v_{1}$ and $u_{1}$ are constructed as above. $\frac{\epsilon}{4}$.

Recall that $\left[w_{1} \mathcal{M}\right]_{2}=L^{2}(\mathcal{D})$, so we can find some $s \in \mathcal{M}$ such that $\left\|w_{1} s-1\right\|_{2} \leq$

The continuity of the maps $x \rightarrow w \rightarrow v$ have already been established in Proposition 1 and Theorem 1 Therefore we can choose some $\delta>0$ so that if $x_{2} \in \mathcal{G}(\mathcal{M}), w_{2}, v_{2}$ and $u_{2}$ are constructed as before, and $\left\|x_{1}-x_{2}\right\|_{\infty}<\delta$, then $\left\|w_{1}-w_{2}\right\|_{2}:\|s\|_{\infty} \leq \frac{\epsilon}{4}$ and $\left\|v_{1}-v_{2}\right\|_{2}:\|s\|_{\infty} \leq \frac{\epsilon}{4}$. Then we have

$$
\begin{aligned}
\left\|u_{1}-u_{2}\right\|_{2} & =\left\|u_{1}-u_{1} w_{1} s-u_{2}+u_{2} w_{1} s+u_{1} w_{1} s-u_{2} w_{2} s+u_{2} w_{2} s-u_{2} w_{1} s\right\|_{2} \\
& \leq 2\left\|1-w_{1} s\right\|_{2}+\left\|v_{1} s-v_{2} s\right\|_{2}+\left\|w_{2} s-w_{1} s\right\|_{2} \\
& \leq 2: \frac{\epsilon}{4}+\frac{\epsilon}{4}+\frac{\epsilon}{4} \\
& =\epsilon . \square
\end{aligned}
$$


Corollary 1. The unitary-analytic factorization for members of $\mathcal{G}(\mathcal{M})$ can be arranged so that the choice of unitaries and the choice of analytics are both continuous as maps from $\left(\mathcal{G}(\mathcal{M}),\|\cdot\|_{\infty}\right)$ to $L^{2}(\mathcal{M})$.

Proof. The result for the unitaries has been established in Theorem 3 . Now if $x_{i}=u_{i} a_{i}$ are such factorizations for $i=1,2$, then the calculation

$$
\begin{aligned}
\left\|a_{1}-a_{2}\right\|_{2} & =\left\|u_{1}^{*} x_{1}-u_{2}^{*} x_{2}\right\|_{2} \\
& =\left\|u_{1}^{*} x_{1}-u_{2}^{*} x_{1}+u_{2}^{*} x_{1}-u_{2}^{*} x_{2}\right\|_{2} \\
& \leq\left\|u_{1}-u_{2}\right\|_{2}:\left\|x_{1}\right\|_{\infty}+\left\|x_{1}-x_{2}\right\|_{\infty}
\end{aligned}
$$

shows that the (obligatory) choice of the analytic component is also continuous.

\section{The Connectivity of $\mathcal{G}\left(H^{\infty}\right)$}

Theorem 4. $\mathcal{G}\left(H^{\infty}\right)$ is connected in the norm of $L^{2}(\mathcal{M})$.

Proof. Suppose $b \in \mathcal{G}\left(H^{\infty}\right)$. We construct a $L^{2}(\mathcal{M})$-continuous path from $b$ to the identity operator.

For $t \in[0,1]$ let $|b|^{t}=u_{t} a_{t}$ be the unitary-analytic factorization examined in $\S 2$

Since $\left|a_{1}\right|=|b|$, by Theorem 2(d) there exists $d \in \mathcal{D}^{u}$ such that $d a_{1}=b$. Now as is well known, an application of Stone's theorem shows that we can find a uniformly continuous path in $\mathcal{D}^{u}$ which connects $d$ to $I$. Since $\mathcal{D}^{u} \subset \mathcal{G}\left(H^{\infty}\right)$, we thus obtain a uniformly continuous path in $\mathcal{G}\left(H^{\infty}\right)$ which connects $b$ to $a_{1}$.

The path in $\mathcal{G}(\mathcal{M})$ given by $\left\{|b|^{t}: t \in[0,1]\right\}$ is uniformly continuous, and so by Corollary 1 the constructed path $\left\{a_{t}: t \in[0,1]\right\}$, connecting $a_{1}$ to $a_{0}$, is continuous in $L^{2}(\mathcal{M})$.

Finally, $|b|^{0}=I=u_{0} a_{0}$, and it is easy to check the constructions of $\$ 2$ ensure that $u_{0}=a_{0}=I$.

Corollary 2. $\mathcal{G}\left(H^{\infty}\right)$ is strongly connected.

Proof. It suffices to note that when we inspect the various paths constructed in Theorem 14 they are all uniformly bounded in the operator norm, and to recall that the strong and $L^{2}(\mathcal{M})$ topologies coincide on bounded sets.

\section{ACKNOWLEDGEMENT}

The authors thank the referee for improving the exposition and pointing out references [4], [5], and [9].

\section{REFERENCES}

[1] T. Ando, Comparison of norms of $\|f(A)-f(B)\|$ and $\|f(|A-B|)\|$, Math. Z. 197 (1988), 403-409. MR 90a:47021

[2] W. Arveson, Analyticity in operator algebras, Amer. J. Math. 89 (1967), 578-642. MR 36:6946

[3] K. Davidson, Nest algebras, Pitman Research Notes in Mathematics Series, vol. 191, Longman Scientific and Technical, 1988. MR 90f:47062

[4] K. Davidson and J. Orr, The invertibles are connected in infinite multiplicity nests, Bull. London Math. Soc. 27 (1995), 155-161. [MR 96c:47062

[5] K. Davidson, J. Orr, and D. Pitts, Connectedness of the invertibles in certain nest algebras, Can. Math. Bull. 38 (1995), 412-420. MR 96m:47088 
[6] P. Dodds and T. Dodds, On a submajorisation inequality of T. Ando, Operator Theory in Function Spaces and Banach Lattices (C.B. Hü̈smans et al., ed.), Operator Theory Advances and Applications, vol. 75, Birkhäuser/Springer-Verlag, Basel/Boston/Berlin, 1995, pp. 113131. MR 96b:47003

[7] T. Fack and H. Kosaki, Generalised s-numbers of $\tau$-measurable operators, Pacific J. Math. 123 (1986), 269-300. MR 87h:46122

[8] R. Kadison and J. Ringrose, Fundamentals of the theory of operator algebras. Volume I: Elementary theory. Volume II: Advanced theory, Pure and Applied Mathematics, Academic Press, London, 1983, 1986. MR 85j:46099; MR 88d:46106

[9] G. Knowles and R. Saeks, On the structure of invertible operators in a nest-subalgebra of a von Neumann algebra, Topics in Operator Theory Systems and Networks (Rehovot, 1983), Operator Theory Advances and Applications, vol. 12, Birkhäuser, Basel/Boston,1984, pp. 303-317. MR 86c: 47064

[10] M. Marsalli, Noncommutative $H^{2}$ spaces, Proc. Amer. Math. Soc. 125 (1997), 779-784. MR 97e:46089

[11] M. Marsalli and G. West, Noncommutative $H^{p}$ spaces, J. Operator Theory 40 (1998), 339355. CMP 99:04

[12] M. McAsey, P. Muhly, and K.-S. Saito, Nonselfadjoint crossed products (invariant subspaces and maximality), Trans. Amer. Math. Soc. 248 (1979), 381-409. MR 80j:46101b

[13] R. Powers and E. Størmer, Free states of the canonical anticommutation relations, Comm. Math. Phys. 16 (1970), 1-33. MR 42:4126

[14] K.-S. Saito, A note on invariant subspaces for finite maximal subdiagonal algebras, Proc. Amer. Math. Soc. 77 (1979), 348-352. MR 81b:46078

Department of Mathematics, Campus Box 4520, Illinois State University, Normal, ILLINOIS 61790-4520

E-mail address: marsalli@math.ilstu.edu

Department of Mathematics, University of the Witwatersrand, 2050 Wits, South AFrica

E-mail address: 036weg@cosmos.wits.ac.za 